\title{
A New Characterization of Compact Sets in Fuzzy Number Spaces
}

\author{
Huan Huang ${ }^{1}$ and Congxin $\mathrm{Wu}^{2}$ \\ ${ }^{1}$ Department of Mathematics, Jimei University, Xiamen 361021, China \\ ${ }^{2}$ Department of Mathematics, Harbin Institute of Technology, Harbin 150001, China
}

Correspondence should be addressed to Huan Huang; hhuangjy@126.com

Received 14 August 2013; Accepted 18 October 2013

Academic Editor: Juan Carlos Cortés López

Copyright (c) $2013 \mathrm{H}$. Huang and C. Wu. This is an open access article distributed under the Creative Commons Attribution License, which permits unrestricted use, distribution, and reproduction in any medium, provided the original work is properly cited.

We give a new characterization of compact subsets of the fuzzy number space equipped with the level convergence topology. Based on this, it is shown that compactness is equivalent to sequential compactness on the fuzzy number space endowed with the level convergence topology. Our results imply that some previous compactness criteria are wrong. A counterexample also is given to validate this judgment.

\section{Introduction}

The convergences on fuzzy number spaces and their applications have been extensively discussed by various authors [113]. One of the most important problems is the characterizations of compact subsets.

Fang and Huang [6] presented a characterization of compact subsets of fuzzy number space endowed with level convergence topology. In this paper, we further give a new characterization of compact subsets of the fuzzy number space equipped with level convergence topology. Based on this, we show that compactness is equivalent to sequential compactness on this type of fuzzy number space.

Diamond and Kloeden [2] presented a characterization of compact sets in fuzzy number spaces equipped with the supremum metric. Fang and Xue [14] also gave a characterization of compact subsets of one-dimensional fuzzy number spaces equipped with the supremum metric. We point out that the compactness criteria given by Fang and Xue are just a special $m=1$ case of the compactness criteria given by Diamond and Kloeden. It is found that there exist contradictions between the characterizations of compact sets given by us and the characterizations given in $[2,14]$. Then it is shown that the characterizations in $[2,14]$ are incorrect by a counterexample.

\section{Fuzzy Number Space}

Let $\mathbb{N}$ be the set of all natural numbers, let $\mathbb{R}^{m}$ be $m$ dimensional Euclidean space, and let $F\left(\mathbb{R}^{m}\right)$ represent all fuzzy subsets on $\mathbb{R}^{m}$, that is, functions from $\mathbb{R}^{m}$ to $[0,1]$. For details, we refer the readers to $[2,12]$.

For $u \in F\left(\mathbb{R}^{m}\right)$, let $[u]_{\alpha}$ denote the $\alpha$-cut of $u$ : that is

$$
[u]_{\alpha}= \begin{cases}\left\{x \in \mathbb{R}^{m}: u(x) \geq \alpha\right\}, & \alpha \in(0,1], \\ \operatorname{supp} u=\overline{\left\{x \in \mathbb{R}^{m}: u(x)>0\right\}}, & \alpha=0 .\end{cases}
$$

We call $u \in F\left(\mathbb{R}^{m}\right)$ a fuzzy number if $u$ has the following properties:

(1) $u$ is normal: there exists at least one $x_{0} \in \mathbb{R}^{m}$ with $u\left(x_{0}\right)=1$

(2) $u$ is convex: $u(\lambda x+(1-\lambda) y) \geq \min \{u(x), u(y)\}$ for $x, y \in \mathbb{R}^{m}$ and $\lambda \in[0,1]$

(3) $u$ is upper semicontinuous;

(4) $[u]_{0}$ is a bounded set in $\mathbb{R}^{m}$.

The set of all fuzzy numbers is denoted by $E^{m}$.

Suppose that $K\left(\mathbb{R}^{m}\right)$ is the set of all nonempty compact sets of $\mathbb{R}^{m}$ and that $K_{c}\left(\mathbb{R}^{m}\right)$ is the set of all nonempty compact 
convex set of $\mathbb{R}^{m}$. The following representation theorem is used widely in the theory of fuzzy numbers.

Proposition 1 (see [15]). Given $u \in E^{m}$, then

(1) $[u]_{1} \neq \emptyset$ and $[u]_{\lambda} \in K_{c}\left(\mathbb{R}^{m}\right)$ for all $\lambda \in[0,1]$;

(2) $[u]_{\lambda}=\bigcap_{\gamma<\lambda}[u]_{\gamma}$ for all $\lambda \in(0,1]$;

(3) $[u]_{0}=\overline{\bigcup_{\gamma>0}[u]_{\gamma}}$.

Moreover, if the family of sets $\left\{v_{\alpha}: \alpha \in[0,1]\right\}$ satisfies conditions (1) through (3), then there exists a unique $u \in E^{m}$ such that $[u]_{\lambda}=v_{\lambda}$ for each $\lambda \in[0,1]$.

Many metrics and topologies on $E^{m}$ are based on the wellknown Hausdorff metric. The Hausdorff metric $H$ on $K\left(\mathbb{R}^{m}\right)$ is defined by

$$
H(U, V)=\max \left\{H^{*}(U, V), H^{*}(V, U)\right\}
$$

for arbitrary $U, V \in K\left(\mathbb{R}^{m}\right)$, where

$$
H^{*}(U, V)=\sup _{u \in U} d(\mathrm{u}, V)=\sup _{u \in U} \inf _{v \in V} d(u, v)
$$

Obviously, if $\left[x_{1}, x_{2}\right]$ and $\left[y_{1}, y_{2}\right]$ are bounded closed intervals of $\mathbb{R}$, then

$$
H\left(\left[x_{1}, x_{2}\right],\left[y_{1}, y_{2}\right]\right)=\max \left\{\left|x_{1}-y_{1}\right|,\left|x_{2}-y_{2}\right|\right\}
$$

Throughout this paper, we suppose that the metric on $\mathbb{R}^{m}$ is the Euclidean metric, and the metric on $K\left(\mathbb{R}^{m}\right)$ is the Hausdorff metric $H$. The Hausdorff metric has the following properties.

Proposition 2 (see $[16,17])$. $(X, d)$ is a metric space, and $K(X)$ is the set of all compact set of $X$. Then

(1) $(X, d)$ complete $\Leftrightarrow(K(X), H)$ complete;

(2) $(X, d)$ separable $\Leftrightarrow(K(X), H)$ separable;

(3) $(X, d)$ compact $\Leftrightarrow(K(X), H)$ compact.

In this paper, we consider two types of convergences on fuzzy number spaces.

(i) Let $u, u_{n} \in E, n=1,2, \ldots$. If $\lim _{n \rightarrow \infty} d_{\infty}\left(u_{n}, u\right)=0$; then we say $\left\{u_{n}\right\}$ supremum converges to $u$, denoted by $u_{n} \stackrel{d_{\infty}}{\longrightarrow} u$, where the supremum metric $d_{\infty}$ is defined by

$$
d_{\infty}(u, v)=\sup _{\alpha \in[0,1]} H\left([u]_{\alpha},[v]_{\alpha}\right)
$$

for all $u, v \in E^{m}$.

(ii) Let $u \in E^{m}$ and let $\left\{u_{\xi}: \xi \in D\right\}$ be a net in $E^{m}$, where $D$ is a direct set. If $\lim _{\xi \in \mathrm{D}} H\left(\left[u_{\xi}\right]_{\alpha},[u]_{\alpha}\right)=0$ for each $\alpha \in[0,1]$, then we say $\left\{u_{\xi}\right\}$ level converges to $u$, denoted by $\lim _{\xi \in D} u_{\xi}=u(l)$ or $u_{\xi} \stackrel{l}{\rightarrow} u$.
Obviously, the supremum metric convergence is stronger than the level convergence on $E^{m}$; that is, if $\left\{u_{n}\right\}$ supremum metric converges to $u$, then it also level converges to $u$.

The symbol $\tau(l)$ is used to denote the topology induced by level convergence on $E^{m}$; that is, $\tau(l)$ is the topology with $\mathscr{B}_{u}=\left\{U_{u}(\alpha, \varepsilon): \varepsilon>0, \alpha \in[0,1]\right\}$ being a local subbase of $u \in E^{m}$, where $U_{u}(\alpha, \varepsilon)=\left\{v \in E^{m}: H\left([u]_{\alpha},[v]_{\alpha}\right)<\varepsilon\right\}$ (see also [6]).

We use $\left(E^{m}, d_{\infty}\right)$ or $\left(E^{m}, \tau(l)\right)$ to denote the fuzzy number space $E^{m}$ equipped with the supremum metric $d_{\infty}$ or equipped with the level convergence topology $\tau(l)$, respectively.

\section{Characterizations of Compact Sets and Sequentially Compact Sets in $\left(E^{m}, \tau(l)\right)$}

In this section, we give characterizations of compact sets and sequentially compact sets, respectively, in $\left(E^{m}, \tau(l)\right)$. Then it is found that compactness is equivalent to sequential compactness on $\left(E^{m}, \tau(l)\right)$. Some propositions and lemmas are needed at first.

Proposition 3 (see [6]). $\left(E^{m}, \tau(l)\right)$ is a Hausdorff space and satisfies the first countability axiom.

Lemma 4. Each compact set of $\left(E^{m}, \tau(l)\right)$ is sequentially compact.

Proof. By Proposition 3, $\left(E^{m}, \tau(l)\right)$ satisfies the first countability axiom, and from the basic topology, every countable compact set of $\left(E^{m}, \tau(l)\right)$ is sequentially compact. Since a compact set is obviously countable compact thus each compact set of $\left(E^{m}, \tau(l)\right)$ is sequentially compact.

A set $S$ is called relatively compact if it has compact closure. A set $U \subset E^{m}$ is said to be uniformly supportbounded if there is a compact set $K \subset \mathbb{R}^{m}$ such that $[u]_{0} \subset K$ for all $u \in U$. Let $\mathscr{F}$ be a family of functions from $S \subset \mathbb{R}$ to $\left(K_{c}\left(\mathbb{R}^{m}\right), H\right)$. Then

(i) $\mathscr{F}$ is said to be equi-left-continuous at $\alpha$ if for each $\varepsilon>$ 0 there exists $\delta(\alpha, \varepsilon)>0$ such that $H\left(f(\alpha), f\left(\alpha^{\prime}\right)\right)<\varepsilon$ whenever $f \in \mathscr{F}$ and $\alpha^{\prime} \in[\alpha-\delta, \alpha]$.

(ii) $\mathscr{F}$ is said to be equi-right-continuous at $\alpha$ if for each $\varepsilon>0$ there exists $\delta(\alpha, \varepsilon)>0$ such that $H\left(f(\alpha), f\left(\alpha^{\prime}\right)\right)<\varepsilon$ whenever $f \in \mathscr{F}$ and $\alpha^{\prime} \in$ $[\alpha, \alpha+\delta]$.

It is said that $\mathscr{F}$ is equi-left (right)-continuous on $S$ if it is equi-left (right)-continuous at each point of $S$.

Note that $[u]$. (where the $\bullet$ may stand for any subscript) can be seen as functions from $[0,1]$ to $K_{c}\left(\mathbb{R}^{m}\right)$. So we can consider whether $\{[u] .: u \in \mathcal{U}\}$ is equi-left (right)-continuous or not for a set $\mathcal{U}$ in $E^{m}$.

Lemma 5. A subset $U$ of $\left(E^{m}, \tau(l)\right)$ is relatively compact if and only if the following conditions are satisfied:

(1) $U$ is uniformly support-bounded.

(2) $\{[u] .: u \in U\}$ is equi-left-continuous on $(0,1]$ and equi-right-continuous at 0 . 
Proof. Necessity. If $U$ is relatively compact in $\left(E^{m}, \tau(l)\right)$, then, by Lemma $4, \bar{U}$ is sequentially compact in $\left(E^{m}, \tau(l)\right)$, and thus $\left\{[u]_{0}: u \in \bar{U}\right\}$ is compact in $K_{c}\left(\mathbb{R}^{m}\right)$. So $\left\{[u]_{0}: u \in \bar{U}\right\}$ is bounded in $K_{c}\left(\mathbb{R}^{m}\right)$; then obviously $U$ is uniformly supportbounded; that is, condition (1) holds.

Now we prove condition (2). In the opposing case where $\left\{[u]_{\alpha}: u \in U\right\}$ is not equi-left-continuous at $\alpha_{0} \in(0,1]$. Then there exist $\varepsilon_{0}>0$ and two sequences $\left\{u_{n}\right\} \subseteq U$ and $\left\{\alpha_{n}\right\} \subseteq$ $(0,1]$ with $\alpha_{n} \rightarrow \alpha_{0}-, n=1,2, \ldots$ such that

$$
H\left(\left[u_{n}\right]_{\alpha_{n}},\left[u_{n}\right]_{\alpha_{0}}\right)>\varepsilon_{0} .
$$

Since $\bar{U}$ is compact, by Lemma $4, \bar{U}$ is sequentially compact. We may assume without loss of generality that $u_{n} \stackrel{l}{\rightarrow} u_{0} \in \bar{U}$. Note that for a given $\beta<\alpha_{0}$, there is an $N$ such that $\alpha_{0}>\alpha_{n}>$ $\beta$ for all $n>N$; hence $\left[u_{n}\right]_{\alpha_{0}} \subseteq\left[u_{n}\right]_{\alpha_{n}} \subseteq\left[u_{n}\right]_{\beta}$ for all $n>N$, and thus by (1)

$$
\begin{aligned}
H\left(\left[u_{0}\right]_{\beta},\left[u_{0}\right]_{\alpha_{0}}\right) & =\lim _{m} H\left(\left[u_{n}\right]_{\beta},\left[u_{n}\right]_{\alpha_{0}}\right) \\
& \geq \lim _{m} H\left(\left[u_{n}\right]_{\alpha_{n}},\left[u_{n}\right]_{\alpha_{0}}\right) \geq \varepsilon_{0}
\end{aligned}
$$

for all $\beta<\alpha_{0}$; this contradicts with $\left[u_{0}\right]_{\alpha_{0}}=\bigcap_{\beta<\alpha_{0}}\left[u_{0}\right]_{\beta}$. Hence $\{[u]$. : $u \in U\}$ is equi-left-continuous on $(0,1]$. Similarly, we can prove that $\{[u] .: u \in U\}$ is equi-rightcontinuous at 0 .

Sufficiency. Notice that $\left(E^{m}, \tau(l)\right)$ can be seen as a subset of the product space $\prod_{\alpha \in[0,1]}\left(K_{c}\left(\mathbb{R}^{m}\right), H\right)$. Let $\bar{U}$ be the closure of $U$ in $\prod_{\alpha \in[0,1]}\left(K_{c}\left(\mathbb{R}^{m}\right), H\right)$. Given $v \in \bar{U}$, there is a net $\left\{u_{\xi}\right.$ : $\xi \in D\}$ of $U$ such that $v=\lim _{\xi \in D} u_{\xi}$. Then obviously

$$
[v]_{\alpha} \in K_{c}\left(\mathbb{R}^{m}\right), \quad[v]_{\mu} \subseteq[v]_{\nu}
$$

for all $\alpha \in[0,1]$ and $\mu \geq \nu$. Given $\gamma \in(0,1]$ and $\varepsilon>0$, from the equi-left-continuity of $\left\{[u]_{.}: u \in U\right\}$ at $\gamma$, there is a $\delta>0$ such that

$$
H\left(\left[u_{\xi}\right]_{\gamma},\left[u_{\xi}\right]_{\gamma-\delta}\right)<\frac{\varepsilon}{3}
$$

for all $\xi \in D$. Since $v=\lim _{\xi \in D} \mathcal{u}_{\xi}$, there exists $k \in D$ such that

$$
H\left([v]_{\gamma},\left[u_{k}\right]_{\gamma}\right)<\frac{\varepsilon}{3}, \quad H\left([v]_{\gamma-\delta},\left[u_{k}\right]_{\gamma-\delta}\right)<\frac{\varepsilon}{3} .
$$

Thus

$$
\begin{aligned}
H\left([v]_{\gamma},[v]_{\gamma-\delta}\right) \leq & H\left([v]_{\gamma},\left[u_{k}\right]_{\gamma}\right)+H\left(\left[u_{k}\right]_{\gamma},\left[u_{k}\right]_{\gamma-\delta}\right) \\
& +H\left(\left[u_{k}\right]_{\gamma-\delta},[v]_{\gamma-\delta}\right)<\varepsilon,
\end{aligned}
$$

and so

$$
\lim _{\delta \rightarrow 0} H\left([v]_{\gamma},[v]_{\gamma-\delta}\right)=0
$$

for all $\gamma \in(0,1]$. Combined with (2) and (3), we know that

$$
[v]_{\alpha}=\bigcap_{\beta<\alpha}[v]_{\beta}
$$

for all $\alpha \in(0,1]$. Similarly, we can prove that

$$
[v]_{0}=\overline{\bigcup_{\beta>0}[v]_{\beta}} .
$$

Then $v \in E^{m}$ from Proposition 1, (2), (13), and (14). So $\bar{U} \subset E^{m}$ from the arbitrariness of $v \in \bar{U}$. This means that the closure of $U$ in $\prod_{\alpha \in[0,1]}\left(K_{c}\left(\mathbb{R}^{m}\right), H\right)$ is just the closure of $U$ in $\left(E^{m}, \tau(l)\right)$. Since $U$ is uniformly support-bounded, then $\left\{[u]_{\alpha}: u \in U\right\}$ is bounded in $\left(K_{c}\left(\mathbb{R}^{m}\right), H\right)$ for each $\alpha \in[0,1]$. By Proposition 2, $\overline{\left\{[u]_{\alpha}: u \in U\right\}}$ is compact in $\left(K_{c}\left(\mathbb{R}^{m}\right), H\right)$ for each $\alpha \in[0,1]$; then from the Tychonoff product theorem $\prod_{\alpha \in[0,1]} \overline{\left.\{u]_{\alpha}: u \in U\right\}}$ is compact in $\prod_{\alpha \in[0,1]}\left(K_{c}\left(\mathbb{R}^{m}\right), H\right)$. So $\bar{U} \quad$ c $\prod_{\alpha \in[0,1]} \overline{\left\{[u]_{\alpha}: u \in U\right\}}$ is compact in $\prod_{\alpha \in[0,1]}\left(K_{c}\left(\mathbb{R}^{m}\right), H\right)$. Since $\bar{U} \subset E^{m}, \bar{U}$ is also a compact set in $\left(E^{m}, \tau(l)\right)$.

Now, we arrive at one of the main results of this section.

Theorem 6. A subset $U$ of $\left(E^{m}, \tau(l)\right)$ is compact if and only if the following conditions are satisfied:

(1) $U$ is closed in $\left(E^{m}, \tau(l)\right)$.

(2) $U$ is uniformly support-bounded.

(3) $\{[u] .: u \in U\}$ is equi-left-continuous on $(0,1]$ and equi-right-continuous at 0.

Proof. Note that $\left(E^{m}, \tau(l)\right)$ is a Hausdorff space, so $U$ is compact if and only if $U$ is closed and relatively compact. The remainder part of proof follows from Lemma 5 immediately.

Fang and Huang [6] proposed a characterization of compact set in $\left(E^{m}, \tau(l)\right)$. They used concepts "eventually equileft-continuous" and "eventually equi-right-continuous."

(i) A net $\left\{u_{k}\right\}_{k \in D}$ in $\left(E^{m}, \tau(l)\right)$ is said to be eventually equi-left-continuous at $\alpha \in(0,1]$, if for each $\varepsilon>$ 0 , there exist a $k_{0} \in D$ and a $\delta>0$ such that $H\left(\left[u_{k}\right]_{\alpha-\delta},\left[u_{k}\right]_{\alpha}\right)<\varepsilon$ for all $k \geq k_{0}$.

(ii) A net $\left\{u_{k}\right\}_{k \in D}$ in $\left(E^{m}, \tau(l)\right)$ is eventually equi-rightcontinuity at $\alpha \in[0,1)$, if for each $\varepsilon>0$, there exist a $k_{0} \in D$ and a $\delta>0$ such that $H\left(\left[u_{k}\right]_{\alpha+\delta},\left[u_{k}\right]_{\alpha}\right)<\varepsilon$ for all $k \geq k_{0}$.

They [6] gave the following compact characterization on $\left(E^{m}, \tau(l)\right)$.

Proposition 7. A closed subset $U$ of $\left(E^{m}, \tau(l)\right)$ is compact if and only if the following conditions are satisfied.

(1) $U$ is uniformly support-bounded.

(2) Each net in $U$ has a subnet which is eventually equileft-continuous on $(0,1]$ and eventually equi-rightcontinuous at 0 .

The readers may compare the condition (3) in Theorem 6 with the condition (2) in Proposition 7. In fact, it can be checked that these two conditions are equivalent. However, 
since the former is a stronger statement in formal, based on it, we can obtain many interesting results. The following Corollary 8 and Theorem 10 are such examples.

Corollary 8. Suppose that $f$ is a continuous function from $[a, b]$ to $\left(E^{m}, \tau(l)\right)$; then $\{[f(x)] .: x \in[a, b]\}$ is equi-leftcontinuous on $(0,1]$ and equi-right-continuous at 0 .

Proof. Since $[a, b]$ is a compact subset of $\mathbb{R}$, we have that $f[a, b]$ is a compact set in $\left(E^{m}, \tau(l)\right)$. The desired result follows immediately from Theorem 6 .

A set $S$ in a topological space is said to be sequentially compact if every sequence in $S$ has a subsequence that converges to a point of $S$ (see also [18]).

Theorem 9. A subset $U$ of $\left(E^{m}, \tau(l)\right)$ is sequentially compact if and only if the following statements are true.

(1) $U$ is closed in $\left(E^{m}, \tau(l)\right)$.

(2) $U$ is uniformly support-bounded.

(3) $\{[u] .: u \in U\}$ is equi-left-continuous on $(0,1]$ and equi-right-continuous at 0.

Proof. Necessity. Given a limit point of $u$ of $U$, since $\left(E^{m}, \tau(l)\right)$ is first countable, there is a sequence $\left\{u_{n}, n=1,2, \ldots\right\}$ of $U$ such that $u=\lim _{n \rightarrow \infty} u_{n}$, and then $u \in U$ according to the sequential compactness of $U$. Thus $U$ is a closed set from the arbitrariness of $u$. So statement (1) holds. Statements (2) and (3) can be proved similarly as in Lemma 5.

Sufficiency. By Theorem 6, if statements (1), (2), and (3) hold, then $U$ is compact, and thus $U$ is sequentially compact from Lemma 4.

The following statement is another main result of this section.

Theorem 10. A subset $U$ of $\left(E^{m}, \tau(l)\right)$ is compact if and only if it is sequentially compact.

Proof. The desired result follows immediately from Theorems 6 and 9.

\section{Applications: To See Some Characterizations of Compact Sets in $\left(E^{m}, d_{\infty}\right)$}

Many authors discussed the characterizations of compact sets in $\left(E^{m}, d_{\infty}\right)$ and obtained many interesting conclusions. However, by using the results in Section 3, it is found that some of those results are incorrect.

Diamond and Kloeden [2, Proposition 8.2.1] have presented the following compactness criteria of sets in $\left(E^{m}, d_{\infty}\right)$.

Theorem 11. A closed set $U$ of $\left(E^{m}, d_{\infty}\right)$ is compact if and only if

(1) $U$ is uniformly support-bounded,
(2) $U^{*}=\left\{u^{*}: u \in U\right\}$ is equi-left-continuous on $[0,1]$ uniformly in $p \in S^{n-1}$ : that is, given $\alpha \in[0,1]$, for each $\varepsilon>0$, there is a $\delta>0$ such that $u^{*}(\alpha, p) \leq u^{*}(\beta, p) \leq$ $u^{*}(\alpha, p)+\varepsilon$ for all $\beta \in[\alpha-\delta, \alpha], p \in S^{n-1}$, and $u \in U$, where the support function $u^{*}:[0,1] \times S^{n-1} \rightarrow \mathbb{R}$ of $u \in E^{m}$ is defined by

$$
u^{*}(\alpha, p)=\sup \left\{\langle p, x\rangle: x \in[u]_{\alpha}\right\} .
$$

Remark 12. Note that

$$
H\left([u]_{\alpha},[v]_{\alpha}\right)=\sup \left\{\left|u^{*}(\alpha, p)-v^{*}(\alpha, p)\right|: p \in S^{n-1}\right\},
$$

so condition (2) in Theorem 11 is equivalent to $\{[u] .: u \in U\}$ is equi-left-continuous on $(0,1]$.

Fang and Xue [14, Theorem 2.3] gave the following characterization of compact subsets in $\left(E^{1}, d_{\infty}\right)$.

Theorem 13. A subset $U$ in $\left(E^{1}, d_{\infty}\right)$ is compact if and only if the following three conditions are satisfied:

(1) $U$ is uniformly support-bounded;

(2) $U$ is a closed subset in $\left(E^{1}, d_{\infty}\right)$;

(3) $\left\{u^{+}(\cdot): u \in U\right\}$ and $\left\{u^{-}(\cdot): u \in U\right\}$ are equi-leftcontinuous on $(0,1]$.

Remark 14. Notice that $[u]_{\alpha}$ is a bounded interval $\left[u^{-}(\alpha), u^{+}(\alpha)\right]$ for all $u \in E^{1}$, so condition (3) holds if and only if $\{[u] .: u \in U\}$ is equi-left-continuous on $(0,1]$. Thus Theorem 13 is just the $m=1$ case of Theorem 11 .

Comparing Theorem 6 with Theorem 11, we can see that there exist conflicts: the supremum metric convergence is stronger than the level convergence on $E^{m}$; however the compactness characterization of $\left(E^{m}, d_{\infty}\right)$ given in Theorem 11 is weaker than that of $\left(E^{m}, \tau(l)\right)$ given in Theorem 6 .

We find that Theorems 11 and 13 are incorrect; the following is a counterexample.

Example 15. Consider a fuzzy number sequence $\left\{u_{n}, n=\right.$ $1,2, \ldots\} \subset E^{1}$ defined by

$$
u_{n}(x)= \begin{cases}1, & x=0, \\ \frac{1}{3}+\frac{2}{3}(1-x)^{n}, & 0<x \leq 1, \quad n=1,2, \ldots, \\ 0, & \text { otherwise. }\end{cases}
$$

Then

$$
\begin{aligned}
& {\left[u_{n}\right]_{\alpha}} \\
& \quad=\left\{\begin{array}{ll}
{\left[0,1-\left(\frac{3}{2} \alpha-\frac{1}{2}\right)^{1 / n}\right],} & \frac{1}{3}<\alpha \leq 1, \\
{[0,1],} & 0 \leq \alpha \leq \frac{1}{3},
\end{array} \quad n=1,2, \ldots,\right.
\end{aligned}
$$

for all $\alpha \in[0,1]$. 
Now we show that $\left\{\left[u_{n}\right]_{,}, n=1,2, \ldots\right\}$ is equi-leftcontinuous on $(0,1]$. In fact, given $\alpha \in(0,1]$, the argument is divided into two cases:

(A) $\alpha \in(1 / 3,1]$. Then for all $\beta \in[(1 / 2)(\alpha+1 / 3), \alpha]$,

$$
\begin{aligned}
H\left(\left[u_{n}\right]_{\alpha},\left[u_{n}\right]_{\beta}\right) \\
\quad=\left(\frac{3}{2} \alpha-\frac{1}{2}\right)^{1 / n}-\left(\frac{3}{2} \beta-\frac{1}{2}\right)^{1 / n} \\
\quad \leq \frac{1}{n}\left(\frac{3}{2} \cdot \frac{1}{2}\left(\alpha+\frac{1}{3}\right)-\frac{1}{2}\right)^{1 / n-1}(\alpha-\beta) \\
\quad \leq\left(\frac{3}{4} \alpha-\frac{1}{4}\right)^{-1}(\alpha-\beta) .
\end{aligned}
$$

So fixed an $\alpha \in(1 / 3,1]$, given $\varepsilon>0$, then

$$
H\left(\left[u_{n}\right]_{\alpha},\left[u_{n}\right]_{\beta}\right) \leq \varepsilon
$$

for all $n \in \mathbb{N}$ and $\beta \in[\alpha-\varepsilon((3 / 4) \alpha-1 / 4), \alpha] \cap[(1 / 2)(\alpha+$ $1 / 3), \alpha]$.

(B) $\alpha \in[0,1 / 3]$. Then for all $\beta \in[0, \alpha]$,

$$
H\left(\left[u_{n}\right]_{\alpha},\left[u_{n}\right]_{\beta}\right)=0 .
$$

Combined with (20) and (21), we know that $\left\{\left[u_{n}\right]_{.}, n=\right.$ $1,2, \ldots\}$ is equi-left-continuous on $(0,1]$.

Let $u$ be a fuzzy number in $E^{1}$ which is defined by

$$
u(x)= \begin{cases}1, & x=0 \\ \frac{1}{3}, & 0<x \leq 1 \\ 0, & \text { otherwise }\end{cases}
$$

Then

$$
[u]_{\alpha}= \begin{cases}\{0\}, & \frac{1}{3}<\alpha \leq 1 \\ {[0,1],} & 0 \leq \alpha \leq \frac{1}{3}\end{cases}
$$

for all $\alpha \in[0,1]$. So

$$
H\left([u]_{\alpha},\left[u_{n}\right]_{\alpha}\right)= \begin{cases}1-\left(\frac{3}{2} \alpha-\frac{1}{2}\right)^{1 / n}, & \frac{1}{3}<\alpha \leq 1, \\ 0, & 0 \leq \alpha \leq \frac{1}{3},\end{cases}
$$

and therefore

$$
d_{\infty}\left(u_{n}, u\right)=\sup \left\{1-\left(\frac{3}{2} \alpha-\frac{1}{2}\right)^{1 / n}: \frac{1}{3}<\alpha \leq 1\right\}=1
$$

for all $n=1,2, \ldots$; thus we know that $d_{\infty}\left(u_{n}, u\right) \nrightarrow 0$.

On the other hand, given $\alpha \in[0,1], H\left(u_{\alpha},\left[u_{n}\right]_{\alpha}\right) \rightarrow$ $0(n \rightarrow \infty)$; hence

$$
u_{n} \stackrel{l}{\longrightarrow} u
$$

It follows from (25) and (26) that $\left\{u_{n}, n=1,2, \ldots\right\}$ has no limit point in $\left(E^{1}, d_{\infty}\right)$. So it is a closed set and is not a compact set in $\left(E^{1}, d_{\infty}\right)$.

Notice that $\left[u_{n}\right]_{0} \subseteq[0,1], n=1,2, \ldots$; then $\left\{u_{n}, n=1,2, \ldots\right\}$ is uniformly support-bounded. Combined with above discussion, we know $\left\{u_{n}, n=1,2, \ldots\right\}$ satisfies conditions (1)-(3) of Theorem 13, and that it is not a compact set in $\left(E^{1}, d_{\infty}\right)$. This shows that Theorem 13 is incorrect.

Remark 16. Fang and Xue [14, Theorem 4.1] give a characterization of compact subsets of all continuous functions from a compact subset $K$ of a metric space $X$ to $\left(E^{1}, d_{\infty}\right)$. However, since it is based on the above theorem, it is wrong too.

\section{Conclusion}

In this paper, we give a characterization of compact sets in fuzzy number space. The result can be used to discuss the analysis properties of fuzzy numbers and fuzzy-numbervalued functions. It can also be used to the applied areas including fuzzy neural networks, fuzzy systems, and so forth.

\section{Conflict of Interests}

The authors declare that there is no conflict of interests regarding the publication of this paper.

\section{Acknowledgment}

This work was supported by the National Science Foundation of China (Grant no. 61103052). The authors would like to thank the reviewers for their invaluable comments and suggestions.

\section{References}

[1] H. Huang and C. Wu, "Approximation capabilities of multilayer fuzzy neural networks on the set of fuzzy-valued functions," Information Sciences, vol. 179, no. 16, pp. 2762-2773, 2009.

[2] P. Diamond and P. Kloeden, Metric Spaces of Fuzzy Sets-Theory and Applications, World Scientific, River Edge, NJ, USA, 1994.

[3] B. M. Ghil, S. Y. Joo, and Y. K. Kim, "A characterization of compact subsets of fuzzy number space," Fuzzy Sets and Systems, vol. 123, no. 2, pp. 191-195, 2001.

[4] O. Kaleva, "On the convergence of fuzzy sets," Fuzzy Sets and Systems, vol. 17, no. 1, pp. 53-65, 1985.

[5] M. Rojas-Medar and H. Román-Flores, "On the equivalence of convergences of fuzzy sets," Fuzzy Sets and Systems, vol. 80, no. 2, pp. 217-224, 1996.

[6] J.-X. Fang and H. Huang, "Some properties of the level convergence topology on fuzzy number space $E^{n}$," Fuzzy Sets and Systems, vol. 140, no. 3, pp. 509-517, 2003, Theme: Topology.

[7] J.-X. Fang and H. Huang, "On the level convergence of a sequence of fuzzy numbers," Fuzzy Sets and Systems, vol. 147, no. 3, pp. 417-435, 2004.

[8] T. Fan, "On the compactness of fuzzy numbers with sendograph metric," Fuzzy Sets and Systems, vol. 143, no. 3, pp. 471-477, 2004. 
[9] G. H. Greco and M. P. Moschen, "Supremum metric and relatively compact sets of fuzzy sets," Nonlinear Analysis. Theory, Methods \& Applications, vol. 64, no. 6, pp. 1325-1335, 2006.

[10] Ö. Talo and F. Başar, "On the slowly decreasing sequences of fuzzy numbers," Abstract and Applied Analysis, vol. 2013, Article ID 891986, 7 pages, 2013.

[11] H. Huang, "Some notes on Zadeh's extensions," Information Sciences, vol. 180, no. 19, pp. 3806-3813, 2010.

[12] C. Wu and M. Ma, The Basic of Fuzzy Analysis, National Defence Industry Press, Beijing, China, 1991, (Chinese).

[13] $\mathrm{H}$. Huang and $\mathrm{C}$. Wu, "Approximation of fuzzy functions by regular fuzzy neural networks," Fuzzy Sets and Systems, vol. 177, pp. 60-79, 2011.

[14] J.-X. Fang and Q.-Y. Xue, "Some properties of the space of fuzzyvalued continuous functions on a compact set," Fuzzy Sets and Systems, vol. 160, no. 11, pp. 1620-1631, 2009.

[15] M. L. Puri and D. A. Ralescu, "The concept of normality for fuzzy random variables," The Annals of Probability, vol. 13, no. 4, pp. 1373-1379, 1985.

[16] E. Klein and A. C. Thompson, Theory of Correspondences, John Wiley \& Sons, New York, NY, USA, 1984.

[17] H. Román-Flores, “The compactness of E(X)," Applied Mathematics Letters, vol. 11, no. 2, pp. 13-17, 1998.

[18] J. L. Kelley, General topology, Springer, New York, NY, USA, 1975. 


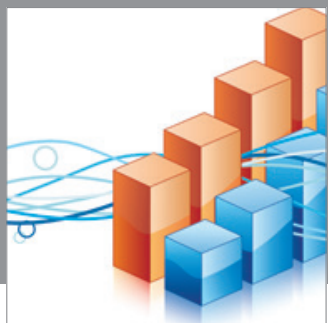

Advances in

Operations Research

mansans

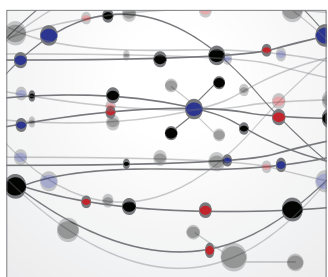

The Scientific World Journal
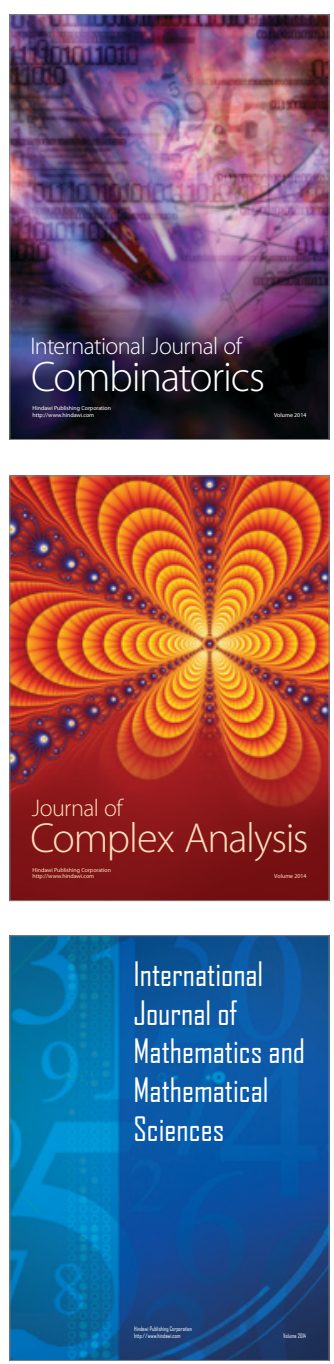
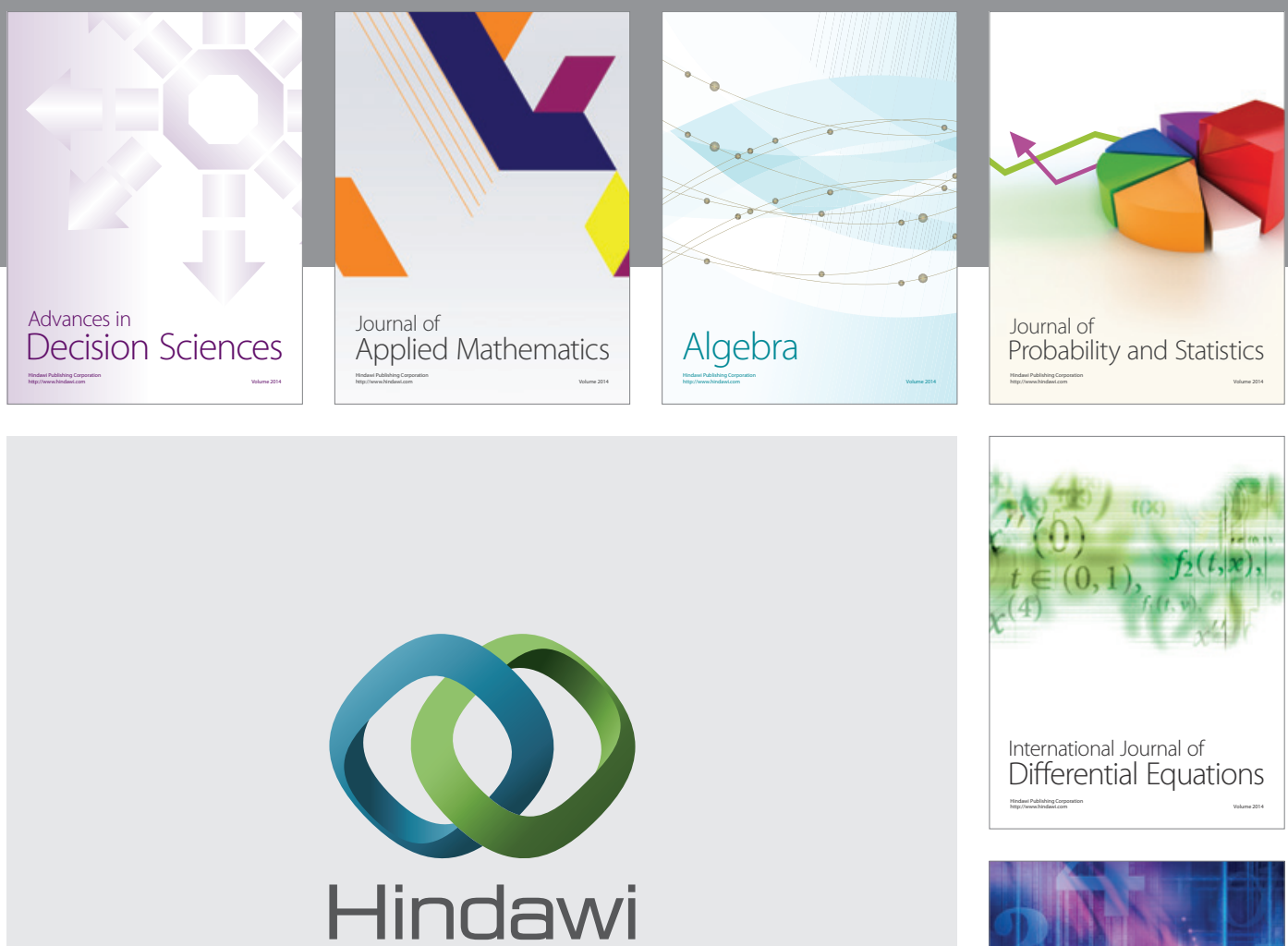

Submit your manuscripts at http://www.hindawi.com
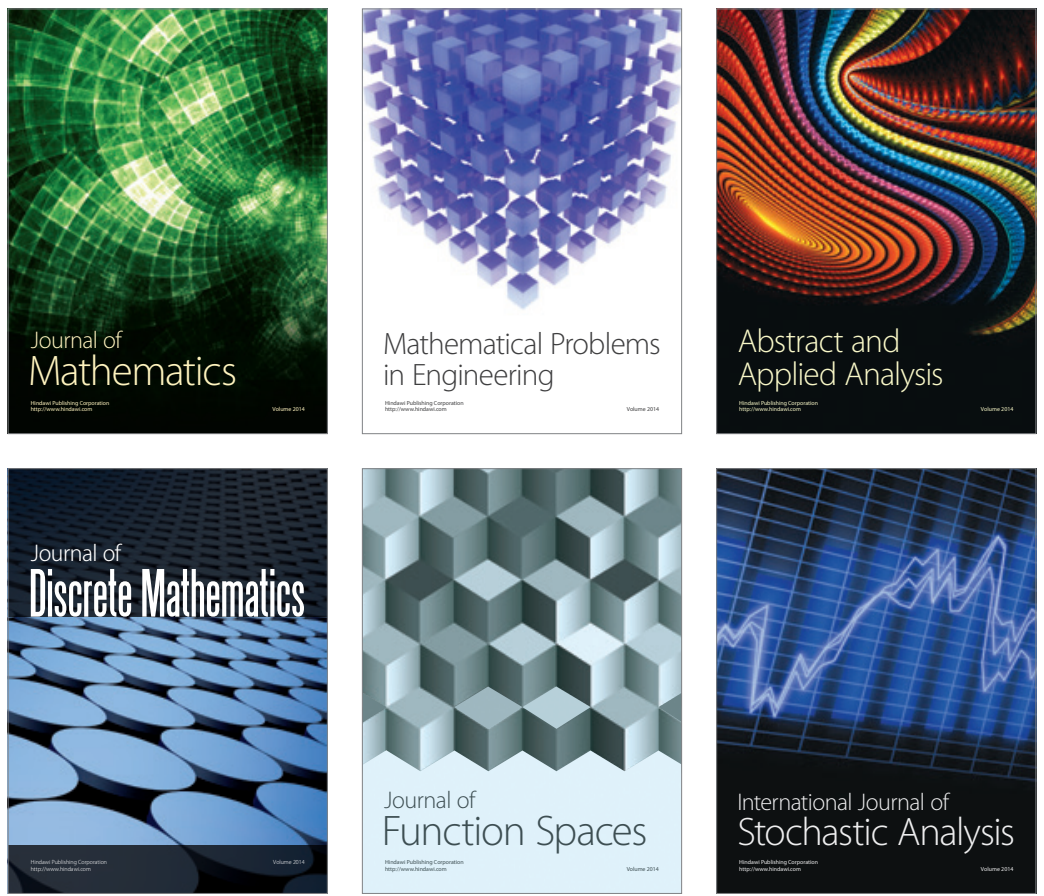

Journal of

Function Spaces

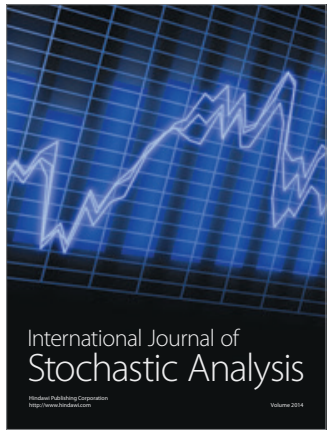

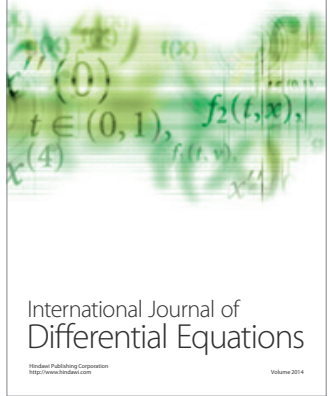
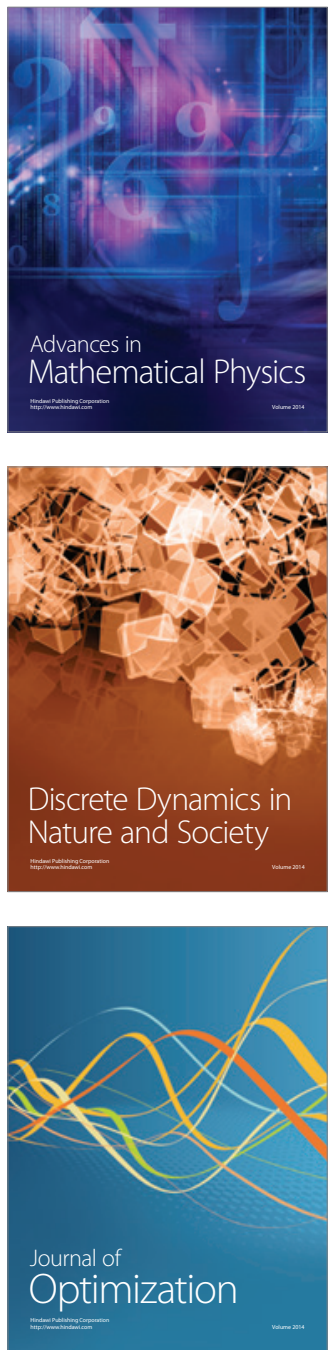